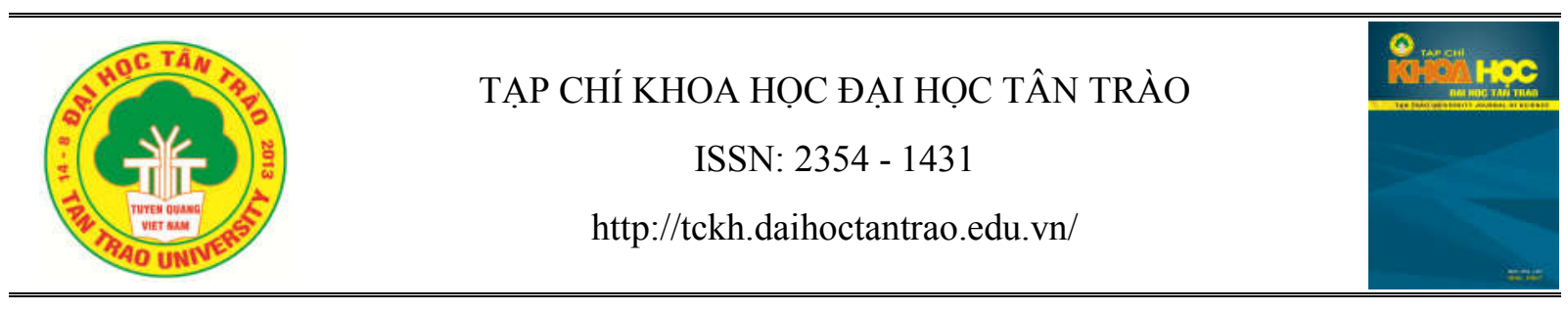

\title{
Sử dụng phần mềm symbolab hỗ trọ̣ dạy học hợp tác môn đại số tuyến tính
}

\author{
Nguyễn Viết Dương ${ }^{a}$, Đinh Thị Hải Bình ${ }^{b}$, Vuoong Thị Hải Hàc ${ }^{c}$, Khổng Chí Nguyện ${ }^{d^{*}}$ \\ ${ }^{a}$ Học viện Công nghệ Buu chính Viễn thông \\ ${ }^{b}$ Trưòng Đại họ Vinh \\ ${ }^{c}$ Truoòng Đại hoc Y Khoa Vinh \\ ${ }^{d}$ Trưòng Đại học Tân Trào \\ *Email:nguyenkc69@gmail.com
}

\section{Thông tin bài viết}

Ngày nhận bài:

$14 / 5 / 2018$

Ngày duyệt đăng:

$10 / 9 / 2018$

\section{Tùr khoá:}

Day hoc hơp tác, phần mềm Symbolab, đại số tuyến tính.

\section{Tóm tắt}

Bài báo đề cập đến việc sử dụng phần mềm Symbolab hỗ trợ dạy học hợp tác (DHHT) môn Đại số tuyến tính. Cụ thể, đó là quan điểm về DHHT; đặc điểm của DHHT; phân biệt giữa dạy học cộng tác và DHHT; quy trình DHHT ở học phần Đại số tuyến tính với sự trợ giúp của phần mềm Symbolab. Phương pháp DHHT là phương pháp dạy học lấy người học làm trung tâm nên đây là phương pháp cần quan tâm nhiều hơn nữa.

\section{Giới thiệu}

Dạy học hợp tác (DHHT) là một trong những xu hướng mới của giáo dục trong thế kỉ XXI, đây cũng là phương pháp dạy học lấy người học làm trung tâm. Phương pháp dạy học này chia toàn bộ sinh viên (SV) của lớp học thành từng nhóm nhỏ. Mỗi nhóm nhỏ chịu trách nhiệm khâu công việc mà mình đảm nhận. Từng cá nhân của nhóm phải tích cực tham gia và chịu trách nhiệm trước cả nhóm. Tất cả đều vì mục đích chung, giải quyết nhiệm vụ chung, hoàn thành công việc được giao. DHHT giống như làm việc trong một dây chuyền sản xuất, nếu một khâu của dây chuyển hỏng thì sản phẩm ra lò sẽ bị lỗi. Mỗi học sinh trong nhóm phải nhận rõ sự thành công hay thất bại của nhóm phụ thuộc vào chính bản thân mình. Các thành viên trao đổi với nhau và hỏi ý kiến nhóm trưởng, thảo luận về nhiệm vụ học tập của mình. Sự nỗ lực của từng thành viên có ý nghĩa vô cùng quan trọng. Từ những nhận định trên ta dễ dàng nhận thấy rằng DHHT là một trong những phương pháp dạy học mang tính tập thể, trong đó có sự hỗ trợ, giúp đỡ lẫn nhau của các cá nhân và kết quả là người học tiếp thu được kiến thức thông qua các hoạt động tương tác giữa người học với người học, giữa người học với giảng viên. Trong giảng dạy đại học, áp dụng phần mềm hỗ trợ giảng dạy sẽ đem lại hiệu quả cao trong giảng dạy nói chung và dạy học theo phương pháp DHHT nói riêng. Ngày nay, cùng với sự phát triển rất nhanh của công nghệ thông tin nhiều phần mềm toán học online được ra đời với giao diện thân thiện gọn nhẹ dễ dàng sử dụng hơn các phần mềm truyền thống. Một trong những phần mềm online ta phải nhắc tới đến đó là phần mềm Symbolab đây là phần mềm hữu hiệu giúp ích cho việc DHHT môn Đại số tuyến tính. Trong khuôn khổ bài báo nhóm tác giả đưa ra phương pháp DHHT môn Đại số tuyến tính với sự trợ giúp cua phần mềm Symbolab online.

\section{Nội dung}

\subsection{Quan điểm về dạy học họp tác}

Theo từ điển tiếng Việt, DHHT là dạy học trong đó người học cùng chung sức, giúp đỡ lẫn nhau trong một công việc, giải quyết nhiệm vụ học tập nhằm đạt được một mục đích chung [2]. Ở đây, người học là các SV đại học, là những đối tượng có nhận thức và ý thức 
tổ chức kỷ luật cao, có tính độc lập, tự chủ, thực sự thuận lợi cho việc tổ chức dạy học hợp tác.

DHHT là dạy học trong đó giáo viên tổ chức và điều khiển các hoạt động học tập hợp tác của nhóm SV, khuyến khích mỗi SV hợp tác tích cực theo khả năng của mỗi người để đạt được những yêu cầu nêu trên của học tập hợp tác, từ đó hình thành kiến thức, kĩ năng mới của bài học [1].

\section{2. Đặc điểm của dạy học hợp tác}

\subsubsection{Sư phu thuộc lẫn nhau tuyệt đối}

Mỗi SV trong cùng một nhóm đóng góp chung cho nỗ lực của toàn nhóm. Các thành viên của nhóm phụ thuộc và dựa vào nhau để đạt được mục tiêu chung. Nỗ lực của mỗi thành viên của nhóm dẫn đến sự thành công của nhóm [3].

\subsubsection{Giải trình cá nhân}

Tất cả các thành viên trong nhóm phải giải trình đóng góp của họ trong công việc, nắm vững công việc họ được giao đối với thành công chung của nhóm [3].

\subsection{3. Đẩy mạnh tuoong tác trục tiếp}

Các nhóm phải đẩy mạnh tương tác cá nhân giữa các thành viên trong nhóm. Các thành viên cung cấp phản hồi, lập luận và kết luận công việc cho các thành viên khác. Giảng viên và nhóm trưởng cần phải là những người khuyến khích, ủng hộ, giúp đỡ các thành viên cùng đạt được mục đích chung của buổi học [3].

2.2.4. Sư dụng các kĩ năng xã hội, giao tiếp cá nhân và làm việc nhóm nhỏ

Các SV được khuyến khích và giúp đỡ để phát triển, rèn luyện tự chịu trách nhiệm, hình thành các kĩ năng kiểm soát mâu thuẫn và quản lí [3].

\subsubsection{Xư lý nhóm}

Các thành viên của nhóm thiết lập mục tiêu nhóm, mô tả những hoạt động mà họ cho là hữu ích hay không hữu ích, công việc của các thành viên và nhóm được giải quyết theo định kì giúp họ nhận ra những thay đổi dù là nhỏ nhất. Những hoạt động này sẽ làm cho từng cá nhân làm việc hiệu quả hơn trong tương lai [3].

\subsection{Phân biệt giữa dạy học cộng tác và dạy học hợp tác}

Theo định nghĩa của từ điển tiếng Việt, dạy học cộng tác là phương pháp dạy học trong đó các SV cùng góp sức làm chung một nhiệm vụ, nhưng có thể không cùng chung một trách nhiệm.

DHHT là phương pháp dạy học trong đó các SV cùng chung sức, giúp đỡ lẫn nhau trong một công việc, giải quyết nhiệm vụ học tập nhằm đạt được một mục đích chung.

Như vậy, dạy học cộng tác và DHHT có chung một điểm đó là cùng góp sức để thực hiện nhiệm vụ dạy học do giảng viên đưa ra, khác nhau là trách nhiệm. Dạy học cộng tác là không cùng chung một trách nhiệm trong khi DHHT là cùng chung một trách nhiệm.

2.4. Quy trình dạy học hợp tác môn Đại số tuyến tính với sự trợ giúp của phần mềm Symbolab

2.4.1. Xác định mục tiêu của việc dạy học họp tác môn Đại số tuyến tính với sụ trọ giúp của phần mềm Symbolab

Mục tiêu đề ra chú trọng đến hoạt động hợp tác của $\mathrm{SV}$, trong đó $\mathrm{SV}$ tương tác với $\mathrm{SV}$, với giảng viên và máy tính, cụ thể ở đây là phần mềm Symbolab để tự kiểm chứng lại lời giải của bài toán. $\mathrm{SV}$ được rèn luyện tư duy phản biện, tư duy sáng tạo, phương pháp giao tiếp và đối thoại với người khác cũng như cách thức giao tiếp giữa $\mathrm{SV}$ và phần mềm Symbolab online.

\subsubsection{Chọn nội dung kiến thức môn Đại số} tuyến tính

DHHT tương đối kén nội dung. Để có thể dạy học kiến thức nào đó theo phương pháp DHHT thì kiến thức đó phải phân chia được thành những kiến thức nhỏ hơn. Những kiến thức nhỏ hơn này là những đơn vị kiến thức phù hợp với từng nhóm đối tượng mà giảng viên phân chia. Những đơn vị kiến thức không quá khó, không quá dài nhưng cũng không quá dễ, không quá ngắn để SV hứng thú trong việc chiếm lĩnh tri thức. Những nhiệm vụ mà giảng viên giao cho từng nhóm SV phải rơi vào "vùng phát triển gần nhất" để $\mathrm{SV}$ cố gắng có thể hoàn thành được công việc mà giảng viên giao phó. Nếu quá dễ thì SV dễ sinh ra tâm lí coi thường kiến thức. Nếu quá khó thì SV dễ chán nản không thích, không hứng thú tham gia bài học. Đối với môn Đại số tuyến tính, giảng viên nên chọn những nội dung kiến thức có nhiều khía cạnh cần giải quyết, nhìn nhận vấn đề theo nhiều cách khác nhau. Chẳng hạn, tổng kết các phương pháp giải bài tập hay tổng kết chương, tìm nhiều cách giải cho một bài toán, tìm quy trình giải một dạng bài tập cụ thể, phát hiện và sửa chữa sai lầm... 


\subsubsection{Thiết kế tiết dạy cụ thể môn Đại số tuyến tính theo phuơng pháp dạy học hợp tác}

Giảng viên giao nhiệm vụ cho SV thông qua phiếu học tập theo cách truyền thống hoặc giao nhiệm vụ cho SV thông qua e-mail, phiếu học tập điện tử hay các trang web xã hội có khả năng tương tác và bảo mật cao (zalo, twitter...) Nhiệm vụ là các kiến thức về phần mềm Symbolab cũng như kiến thức về Đại số tuyến tính sẽ được thảo luận, để SV có thời gian chuẩn bị trước khi lên lớp. Đó có thể là các cách giải khác nhau, cách thức phát hiện và giải quyết vấn đề, dự đoán về những mâu thuẫn khác nhau trong hợp tác nhóm, phương pháp thảo luận nhóm, cách thức thống nhất chung của cả nhóm.

\subsubsection{Tổ chức hoạt động hợp tác của lớp}

Giảng viên tốt ngoài biết cách phân chia thành các đơn vị kiến thức thì còn phải biết phân lớp thành các nhóm phù hợp với ý đồ tổ chức DHHT của giảng viên. Vì là phương pháp DHHT nên trong từng nhóm thì nhóm trưởng là người quan trọng. Đây là "tổng công trình sư” kết nối tất cả mọi người trong nhóm lại với nhau. Nhóm trưởng có thể không phải là người giỏi chuyên môn nhất nhưng là người biết phát huy ưu điểm, hạn chế nhược điểm của từng thành viên nhóm. Biết phát huy, sáng tạo công việc vì mục đích chung. Có nhiều cách chia nhóm khác nhau. Giảng viên có thể chia lớp thành các nhóm đôi, nhóm $5-6$ học sinh, nhóm có đầy đủ các trình độ hay nhóm chỉ có một loại trình độ. Ngoài việc biết phân chia nhóm thì giảng viên còn phải biết tổ chức hình thức học thi đua giữa các nhóm. Giảng viên tạo cơ hội để cho các nhóm thảo luận, hợp tác với nhau. Giảng viên phải biết đánh giá từng cá nhân và tập thể một cách công bằng, khách quan.

\subsection{Ví dụ minh họa}

Giảng viên chia lớp thành 3 nhóm: Nhóm sinh viên trung bình (SVB), nhóm sinh viên khá (SVK) và nhóm sinh viên giỏi (SVG). Các nhóm đều được sử dụng phần mềm Symbolab trong giải toán. Các nhiệm vụ được giảng viên chiếu lên bảng bằng máy chiếu.

Đối với nhóm SVB, giảng viên giao nhiệm vụ tính định thức:

Bài toán 1.1 Tính định thức:

$$
A_{1}=\left|\begin{array}{lll}
2 & 1 & 1 \\
1 & 2 & 1 \\
1 & 1 & 2
\end{array}\right| \text { ? }
$$

Đối với nhóm SVK, giảng viên giao nhiệm vụ tính định thức:

Bài toán 1.2 Tính định thức

$A_{2}=\left|\begin{array}{ccc}1+x & 1 & 1 \\ 1 & 1+x & 1 \\ 1 & 1 & 1+x\end{array}\right| ?$

Đối với nhóm SVG, giảng viên giao nhiệm vụ tính định thức:

Bài toán 1.3 Tính định thức

$$
A_{3}=\left|\begin{array}{ccc}
1+x & x & x \\
x & 1+x & x \\
x & x & 1+x
\end{array}\right| ?
$$

Qua việc giao nhiệm vụ cho ba nhóm với năng lực riêng biệt giảng viên nhận thấy ba nhóm SV hứng thú với nhiệm vụ mà mình được giao.

- Đối với nhóm SVB, đa số các em đều hoàn thành việc tính định thức. Một số em học lực yếu hơn thì sử dụng phần mềm Symbolab sẽ giúp các em tìm được kết quả đúng của định thức $A_{1}=4$. Ngay trên kết quả phần mềm Symbolab cũng nhắc lại lý thuyết một cách rõ ràng để $\mathrm{SV}$ yếu hơn nhớ lại được cách tính định thức cấp 4 và thao tác trên phần mềm một cách chính xác.

Bước 1: Truy cập vào địa chỉ https://www.symbolab.com

Bước 2: Trên ô cửa sổ lệnh nhập chức năng tính định thức và chọn kích thước của mà trận như hình sau:

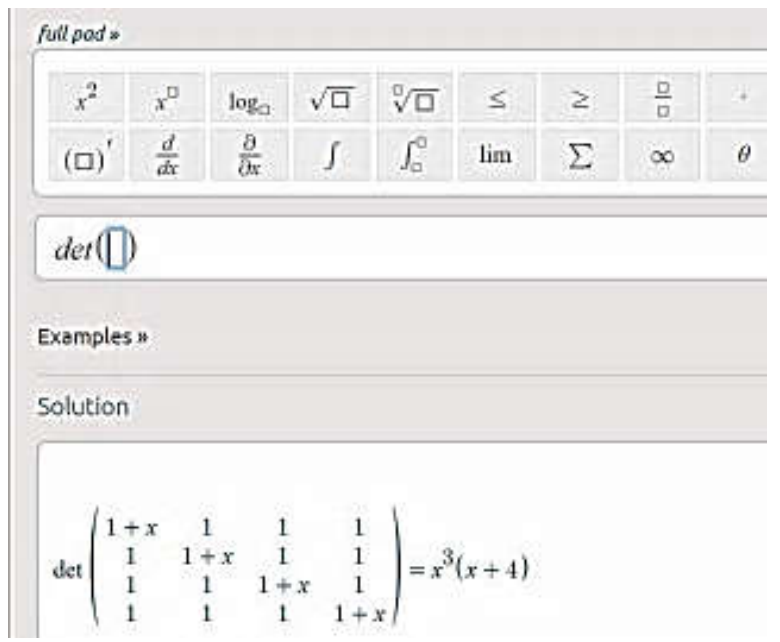

Bước 3: Nhập số liệu của các phần tử của ma trận rồi chọn phím "Enter" như ví dụ trên thì ta được kết quả như sau: 


$$
\begin{aligned}
& \operatorname{det}\left(\begin{array}{lll}
2 & 1 & 1 \\
1 & 2 & 1 \\
1 & 1 & 2
\end{array}\right)=4 \\
& \text { Steps } \\
& \operatorname{det}\left(\begin{array}{lll}
2 & 1 & 1 \\
1 & 2 & 1 \\
1 & 1 & 2
\end{array}\right)
\end{aligned}
$$

Find the matrix determinant according to formula:

$\operatorname{det}\left(\begin{array}{lll}a & b & c \\ d & e & f \\ g & h & i\end{array}\right)=a \cdot \operatorname{det}\left(\begin{array}{ll}e & f \\ h & i\end{array}\right)-b \cdot \operatorname{det}\left(\begin{array}{ll}d & f \\ g & i\end{array}\right)+c \cdot \operatorname{det}\left(\begin{array}{ll}d & e \\ g & h\end{array}\right)$

$=2 \cdot \operatorname{det}\left(\begin{array}{ll}2 & 1 \\ 1 & 2\end{array}\right)-1 \cdot \operatorname{det}\left(\begin{array}{ll}1 & 1 \\ 1 & 2\end{array}\right)+1 \cdot \operatorname{det}\left(\begin{array}{ll}1 & 2 \\ 1 & 1\end{array}\right)$

- Đối với nhóm SVK, thì các em đều thực hiện được việc tính định thức $A_{2}$ bằng quy tắc Sarrus.

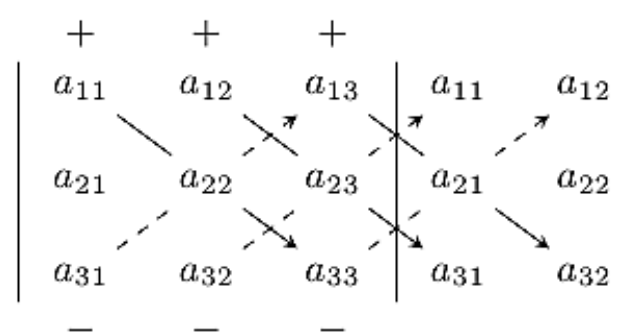

Một số em SVK còn giải bài toán này bằng nhiều cách khác nhau. Có em sử dụng định nghĩa định thức để tính, có em sử dụng phương pháp biến đổi Gauss, có em sử dụng phần mềm Symbolab cho ra lời giải để tự kiểm chứng kết quả của mình. Kết quả các em đưa ra là $A_{2}=x^{3}+3 x^{2}$.

- Đối với nhóm SVG thì các em đều giải được bài toán. Đa phần các em đều giải được bằng ba phương pháp khác nhau, đó là phương pháp biến đổi ma trận đưa về ma trận tam giác, phương pháp Sarrus, phương pháp định nghĩa.

- Giảng viên yêu cầu các em SVG tìm thêm một số cách giải khác ba cách này. Một em vốn là học sinh chuyên ở bậc phổ thông đã đưa ra cách giải “đẹp” sau:

$$
\begin{aligned}
& A_{3}=\left|\begin{array}{ccc}
1+x & x & x \\
x & 1+x & x \\
x & x & 1+x
\end{array}\right|=\left|\begin{array}{ccc}
1+3 x & x & x \\
1+3 x & 1+x & x \\
1+3 x & x & 1+x
\end{array}\right| \\
& =(1+3 x)\left|\begin{array}{ccc}
1 & x & x \\
1 & 1+x & x \\
1 & x & 1+x
\end{array}\right|=(1+3 x)\left|\begin{array}{ccc}
1 & x & x \\
0 & 1 & x \\
0 & 0 & 1
\end{array}\right|=1+3 x .
\end{aligned}
$$

Để kiểm tra lại kết quả, giảng viên yêu cầu sinh viên dùng phần mềm Symbolab để kiểm tra được kết quả thể hiện trên màn hình như sau:

$$
\begin{aligned}
& \operatorname{det}\left(\left(\begin{array}{ccc}
1+x & 1 & 1 \\
1 & 1+x & 1 \\
1 & 1 & 1+x
\end{array}\right)\right) \\
& \text { Examples " } \\
& \text { Solution } \\
& \operatorname{det}\left(\begin{array}{ccc}
1+x & 1 & 1 \\
1 & 1+x & 1 \\
1 & 1 & 1+x
\end{array}\right)=x^{3}+3 x^{2} \\
& \text { Steps } \\
& \operatorname{det}\left(\begin{array}{ccc}
1+x & 1 & 1 \\
1 & 1+x & 1 \\
1 & 1 & 1+x
\end{array}\right)
\end{aligned}
$$

- Giảng viên yêu cầu các SV nhận xét về mối quan hệ giữa 3 bài toán 1.1, 1.2, 1.3. Giảng viên ưu tiên sự phát biểu của các em SVB.

- SVB: Bài toán 1.2 và 1.3 là bài toán tổng quát của bài toán 1.1 . Bài toán 1.2 và 1.3 là hai bài toán tương tự nhau.

- Giảng viên yêu cầu SVB mở rộng bài toán 1.1, SVK mở rộng bài toán $1.2, \mathrm{SVG}$ mở rộng bài toán 1.3 từ định thức cấp 3 , thành định thức cấp 4 . Kết quả giảng viên thu được các bài toán sau:

Bài toán 2.1 Tính định thức

$$
B_{1}=\left|\begin{array}{llll}
2 & 1 & 1 & 1 \\
1 & 2 & 1 & 1 \\
1 & 1 & 2 & 1 \\
1 & 1 & 1 & 2
\end{array}\right| ?
$$

Bài toán 2.2 Tính định thức

$$
B_{2}=\left|\begin{array}{cccc}
1+x & 1 & 1 & 1 \\
1 & 1+x & 1 & 1 \\
1 & 1 & 1+x & 1 \\
1 & 1 & 1 & 1+x
\end{array}\right| ?
$$

Bài toán 2.3 Tính định thức

$$
B_{3}=\left|\begin{array}{cccc}
1+x & x & x & x \\
x & 1+x & x & x \\
x & x & 1+x & x \\
x & x & x & 1+x
\end{array}\right| ?
$$

Qua quan sát, giảng viên nhận thấy có một số em SVB sử dụng phương pháp tương tự quy tắc Sarrus cho định thức cấp 3 để tính định thức cấp 4. 


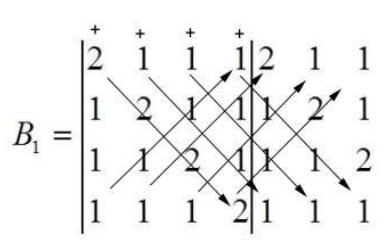

Cách làm này cho ta kết quả $\operatorname{det}\left(B_{1}\right)=9$.

- Giảng viên cho các SV khác phát biểu xem đây là lời giải đúng hay sai?

- Qua quan sát, các SV còn lại đều cho đây là kết quả sai, bởi tính bằng các phương pháp khác hay sử dụng phần mềm Symbolab cho kết quả hiện trên phần mềm như sau:

$$
\operatorname{det}\left(\begin{array}{cccc}
2 & 1 & 1 & 1 \\
1 & 2 & 1 & 1 \\
1 & 1 & 2 & 1 \\
1 & 1 & 1 & 2
\end{array}\right)
$$

Examples "

Solution

$\operatorname{det}\left(\begin{array}{llll}2 & 1 & 1 & 1 \\ 1 & 2 & 1 & 1 \\ 1 & 1 & 2 & 1 \\ 1 & 1 & 1 & 2\end{array}\right)=5$

Steps

$\operatorname{det}\left(\begin{array}{llll}2 & 1 & 1 & 1 \\ 1 & 2 & 1 & 1 \\ 1 & 1 & 2 & 1 \\ 1 & 1 & 1 & 2\end{array}\right)$

Sinh viên sẽ không giải thích được tại sao kết quả lại khác so với phần mềm. Khi đó, giảng viên mới chốt lại kiến thức. Thực ra, người ta đã tìm được quy tắc Sarrus cho định thức cấp 4 nhưng "không đẹp" bằng định thức cấp 3. Quy tắc Sarrus cho định thức cấp 4 như sau:

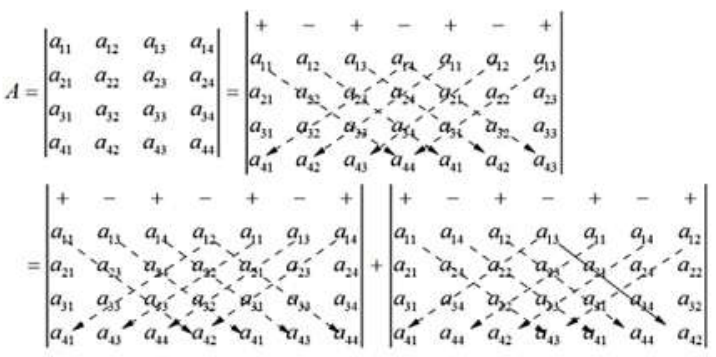

Giảng viên để sinh viên tính toán đối với các Ví dụ 2.2 và 2.3 trên phần mềm Symbolab để kiểm tra lại lý thuyết. Sau khi thao tác trên máy $\mathrm{SV}$ có được kết quả của hai ví dụ trên màn hình như sau:

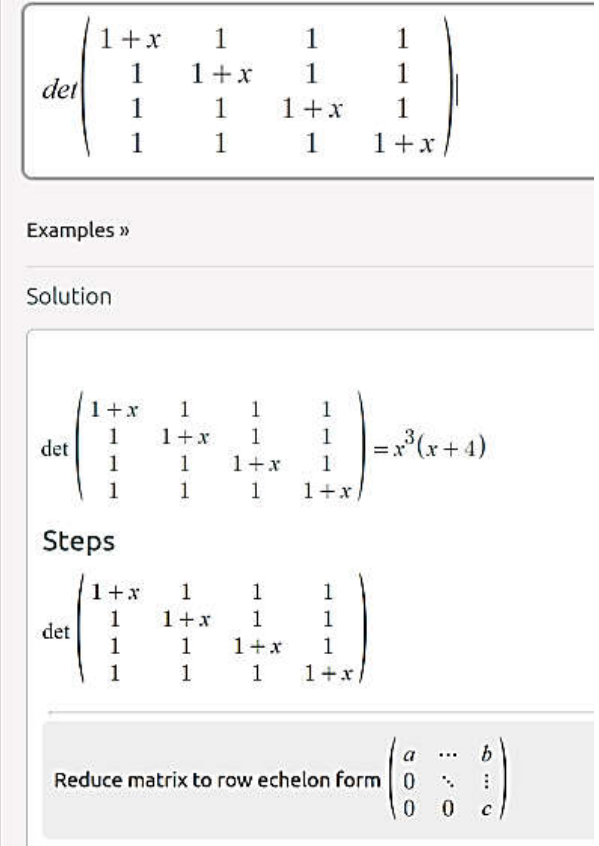

Đối với Ví dụ 2.3 thao tác trên phần mềm tương tự SV sẽ được kết quả. Từ kết quả trên phần mềm giúp $\mathrm{SV}$ củng cố kiến thức và nhớ được kiến thức.

- Giảng viên yêu cầu các em $\mathrm{SVB}, \mathrm{SVK}$ và $\mathrm{SVG}$ phát biểu bài toán tổng quát của các bài toán $2.1,2.2$ và 2.3 như sau:

Bài toán 3.1 Tính định thức:

$C_{1}=\left|\begin{array}{cccc}2 & 1 & \ldots & 1 \\ 1 & 2 & \ldots & 1 \\ \ldots & \ldots & \ldots & \ldots \\ 1 & 1 & \ldots & 2\end{array}\right| ?$

Bài toán 3.2 Tính định thức:

$C_{2}=\left|\begin{array}{cccc}1+a_{1} & a_{2} & \ldots & a_{n} \\ a_{1} & 1+a_{2} & \ldots & a_{n} \\ \ldots & \ldots & \ldots & \ldots \\ a_{1} & a_{2} & \ldots & 1+a_{n}\end{array}\right| ?$

Bài toán 3.3 Tính định thức

$C_{3}=\left|\begin{array}{cccc}a & x & \ldots & x \\ x & a & \ldots & x \\ \ldots & \ldots & \ldots & \ldots \\ x & x & \ldots & a\end{array}\right| ?$

- Giảng viên yêu cầu các em $\mathrm{SVK}$ và $\mathrm{SVG}$ cùng tính các bài toán 3.2 và 3.3. Một số em $\mathrm{SV}$ sử dụng cách giải tương tự cách giải bài toán 1.3 tính được các kết quả như sau. Đối với bài toán 3.2: 


\section{Cách 1.}

$$
\begin{aligned}
& C_{2}=\left|\begin{array}{cccc}
1+a_{1} & a_{2} & \ldots & a_{n} \\
a_{1} & 1+a_{2} & \ldots & a_{n} \\
\ldots & \ldots & \ldots & \ldots \\
a_{1} & a_{2} & \ldots & 1+a_{n}
\end{array}\right| \\
& \left|\begin{array}{cccc}
1+a_{1}+\ldots+a_{n} & a_{2} & \ldots & a_{n} \\
1+a_{1}+\ldots+a_{n} & 1+a_{2} & \ldots & a_{n} \\
\ldots & \ldots & \ldots & \ldots \\
1+a_{1}+\ldots+a_{n} & a_{2} & \ldots & 1+a_{n}
\end{array}\right| \\
& =\left(1+a_{1}+\ldots+a_{n}\right)\left|\begin{array}{cccc}
1 & a_{2} & \ldots & a_{n} \\
1 & 1+a_{2} & \ldots & a_{n} \\
1 & \ldots & \ldots & \ldots \\
1 & a_{2} & \ldots & 1+a_{n}
\end{array}\right| \\
& =\left(1+a_{1}+\ldots+a_{n}\right)\left|\begin{array}{cccc}
1 & a_{2} & \ldots & a_{n} \\
0 & 1 & \ldots & a_{n} \\
\ldots & \ldots & \ldots & \ldots \\
0 & 0 & \ldots & 1
\end{array}\right| \\
& =1+a_{1}+\ldots+a_{n} .
\end{aligned}
$$

Cách 2.Xem lời giải tương tự bài toán 3.3.

Đối với bài toán 3.3 , ta có các cách giải sau:

\section{Cách 1.}

$$
\begin{aligned}
& C_{3}=\left|\begin{array}{cccc}
a-x+x & x & \ldots & x \\
0+x & a & \ldots & x \\
\ldots & \ldots & \ldots & x \\
0+x & x & \ldots & a
\end{array}\right|=\left|\begin{array}{cccc}
a-x & x & \ldots & x \\
0 & a & \ldots & x \\
\ldots & \ldots & \ldots & x \\
0 & x & \ldots & a
\end{array}\right| \\
& +x\left|\begin{array}{cccc}
1 & x & \ldots & x \\
1 & a & \ldots & x \\
\ldots & \ldots & \ldots & x \\
1 & x & \ldots & a
\end{array}\right|=(a-x) P_{n-1}+x Q_{n-1} \\
& x Q_{n-1}=x(a-x)^{n-1} \\
& P_{n-1}=(a-x) P_{n-2}+x Q_{n-2} \\
& \Rightarrow C_{3}=(a-x)^{2} P_{n-2}+x(a-x) Q_{n-2} \\
& =(a-x)^{2} P_{n-2}+x(a-x)^{n-1} \text {; } \\
& C_{3}=P_{2}+x(a-x)^{n-1}+\ldots+x(a-x)^{n-1} .
\end{aligned}
$$

Vậy

$$
\mid \begin{aligned}
& C_{3}=a(a-x)^{n-1}+x(a-x)^{n-1}+\ldots+x(a-x)^{n-1} \\
& \frac{1=a(a-x)^{n-1}+(n-1) x(a-x)^{n-1}}{\quad} \\
& =((n-1) x+a)(a-x)^{n-1} .
\end{aligned}
$$

\section{Cách 2.}

Tương tự cách 1 bài toán 3.2 .
- Giảng viên tổng kết kết quả thi đua, có chấm điểm cụ thể (điểm tối đa cho mỗi nhóm là 10 điểm, bao gồm giải toán đúng là 3 điểm, tìm được nhiều cách giải: 3 điểm ( 3 cách, mỗi cách 1 điểm), mở rộng bài toán: 3 điểm, phát hiện lỗi sai 1 điểm).

- Giảng viên nhận xét cách học của cả nhóm, rút kinh nghiệm phương pháp tổ chức, tác phong học tập của nhóm, của từng cá nhân. Nêu ưu điểm, nhược điểm và tuyên dương cá nhân học tập tốt. Nhận xét khuyết điểm của cách DHHT từ đó đề xuất biện pháp khắc phục để lần sau học tốt hơn.

\section{Kết luận}

Khi thực hiện việc giảng dạy bài giảng này trên thực tế, cụ thể là ở Học viện Công nghệ Bưu chính Viễn thông, cơ sở TP. Hồ Chí Minh, Đại học Y Khoa Vinh, Đại học Tân Trào, chúng tôi nhận thấy, tất cả các $\mathrm{SV}$ đều hứng thú với việc sử dụng phần mềm Symbolab để tính toán định thức. Sinh viên nhận thấy rằng dễ dàng giải quyết được các bài toán mà giảng viên đưa ra. Sau khi mở rộng định thức sang cấp 4 thì một số em đã quen đường mòn nếp cũ, áp dụng quy tắc Sarrus một cách máy móc, không đúng bản chất của quy tắc Sarrus. Giảng viên phải chốt lại kiến thức để SV không mắc phải sai lầm. Sau khi thảo luận, các em đều nhận thấy sai lầm và hiểu được vấn đề. Đặc biệt đã có sự tiến bộ rõ rệt, nhiều em SVB khi thi giữa kì đã đạt được điểm khá, một số em SVK khá đạt điểm giỏi và một số em SVG đạt điểm xuất sắc. Mặc dù phương pháp DHHT có nhiều hạn chế như thời gian hạn chế, năng lực của từng $\mathrm{SV}$ không đồng đều, năng lực công nghệ thông tin, cụ thể là năng lực sử dụng phần mềm Symbolab ở một số em còn chưa thành thạo. Việc phát hiện sai lầm và sửa chữa sai lầm còn có hạn chế nhưng phương pháp DHHT rõ ràng là một phương pháp dạy học tốt, kích thích người học. Người học được hoạt động trong giao lưu, trong tương tác. Các SV gắn bó với nhau mật thiết, tạo mối liên kết chặt chẽ hơn so với cách dạy học truyền thống. SV được đào tạo các hầu hết các năng lực quan trọng, cốt lõi mà một $\mathrm{SV}$ cần có. Đó là năng lực phát hiện và giải quyết vấn đề, năng lực công nghệ thông tin, năng lực lập luận toán học, năng lực tư duy toán học, năng lực thẩm mĩ, năng lực biểu diễn toán học và cái cốt yếu của cách dạy này đó là năng lực giao tiếp toán học. 


\section{TÀI LIỆU THAM KHẢO}

1. Trần Ngọc Lan, Vũ Thị Minh Hằng (2005), Áp dụng DHHT trong dạy học toán ở tiểu học, Tạp chí Giáo dục;

2. Hoàng Phê (1988), Tù điển tiếng Việt, Nxb Khoa học xã hội;

3.Https://www.csuchico.edu/ourdemocracy/_assets/do cuments/pedagogy/li,-m.-p.-_-lam,-b.-h.-2013-

cooperative-learning.pdf;

4. Hoàng Lê Minh (2007), Thiết kế tình huống hoạt động hoc tập hơp tác trong dạy học môn toán, Tạp chí Giáo dục;
5. Bernd Meier, Nguyễn Văn Cường (2005), "Phát triển năng lực thông qua phuoong pháp và phuoong tiện dạy học mới", Tài liệu hội thảo tập huấn, Dự án phát triển giáo dục THPT, Hà Nội;

6. David W.Johnson, Roger T.Johnson, Holubec (1994), "Cooperative Learning in The Classroom", Association For Supervision and Curriculum Development Alexandria Virgnia;

7. Elizabeth G.Cohen, Cleste M. Brody, Mara Sapon Shevin (2004), Teaching Cooperative Learning, State University of New York Press, Albany.

\section{Using the symbolab online software to aid the cooperative learning of linear algebra} Nguyen Viet Duong, Dinh Thi Hai Binh, Vuong Thi Hai Ha, Khong Chi Nguyen

\section{Article info}

Recieved:

14/5/2018

Accepted:

10/9/2018

Keywords:

Cooperative learning, symbolab software, linear algebra.

\begin{abstract}
The article examinesthe applications of the Symbolab software to aid the cooperative learning in teaching linear algebra. Particularly, that is the point of view of cooperative learning, the characteristics of cooperative learning, the difference between collaborative learning and cooperative learning, and the processing of cooperative learning of linear algebra with the help of the Symbolab software. The cooperative learning is student-centered one, and thus we should pay more attention in using this method.
\end{abstract}

